\title{
Stress Monitoring in Steel Elements via Detection of AC Magnetic Permeability Changes
}

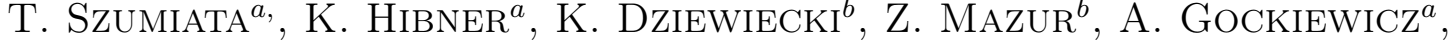 \\ M. Gzik-Szumiata ${ }^{a}$, B. Górka ${ }^{a}$, M. Witośs ${ }^{c *}$ \\ ${ }^{a}$ Department of Physics, University of Technology and Humanities in Radom, Krasickiego 54, 26-600 Radom, Poland \\ ${ }^{b}$ Institute of Applied Mechanics and Power Engineering, University of Technology and Humanities in Radom, \\ Krasickiego 54, 26-600 Radom, Poland \\ ${ }^{c}$ Air Force Institute of Technology, Księcia Bolesława 6, 01-494 Warsaw, Poland
}

\begin{abstract}
The influence of mechanical stress on low frequency AC magnetic permeability was studied. The cold-drawn bars with $\mathrm{C} 45$ steel were subjected to investigation. The tensile stress (within elastic regime) was applied by means of material testing machine. Simple measuring system was assembled, which consisted of: function generator with magnetizing coil, detection coil and precise AC voltmeter. The registered changes of induced voltage were proportional to the change of magnetic permeability of the stretched rods. The obtained results were almost frequency-independent due to low frequency limit (250-500 Hz, weak eddy currents, no spin-origin energy dissipation). A significant magneto-mechanical hysteresis was observed slightly evolving from cycle to cycle with tendency of stabilization. The extension of basic Stoner-Wohlfarth model of magnetic permeability allowed to fit the data reproducing hysteretic behavior and considering the relaxation of the internal stress. The proposed, low-cost method is suitable in the industrial applications for stress control in large-sized steel elements.
\end{abstract}

DOI: $10.12693 /$ APhysPolA.133.719

PACS/topics: 75.80.+q, 75.60.Ej, 75.50.Bb, 75.30.Gw, 62.20.D-

\section{Introduction}

The phenomenon of stress/strain-induced changes of magnetization (and other magnetic parameters) was discovered in $19^{\text {th }}$ century by Villari [1] and is usually called the inverse magnetostrictive effect. The source of magnetostriction is strain dependence of magneto-crystalline anisotropy originating from the spin-orbital coupling of electrons. The variety of magneto-mechanical effects in bulk material and thin films was reported both in historical papers $[2,3]$ and in more recent ones [4-9]. These phenomena can be utilized for determining magnetoelastic parameters of materials [8] as well as for nondestructive evaluation (NDE) of mechanical elements [7]. The static (DC) magneto-mechanical phenomena can be effectively described by Jiles-Atherton-Sablik model of magnetic hysteresis [9-11] in medium and high magnetic fields.

The main goal of present work was to observe and to model the changes of AC magnetic permeability of steel bars caused by tensile stress. The motivation of this research was the deficiency of works devoted to AC, lowfield magneto-mechanical effects as well as a will of verification whether they can be practically utilized for stress monitoring.

\section{Experimental setup}

The object of investigations were commonly available, cold-drawn bars of $\phi=12 \mathrm{~mm}$ diameter made with

*corresponding author; e-mail: t.szumiata@uthrad.pl non-alloy, medium-carbon, quality steel C45 (1.0503). Bars were put in material testing machine producing tensile stress up to $300 \mathrm{MPa}$ - near upper yield strength ( $R e \approx 305 \mathrm{MPa}$ for $\phi<16 \mathrm{~mm}$ ), pointing the elastic linearity limit. The standard testing machine was equipped with dedicated magnetometric system consisting of function generator, magnetizing coil, signal coil, high quality AC voltmeter. Stretched bar piece (about $1 \mathrm{~m}$ long) was placed inside both coils separated by nonmagnetic cylinder (about $20 \mathrm{~cm}$ high). The amplitude of magnetizing $\mathrm{AC}$ field did not exceed $800 \mathrm{~A} / \mathrm{m}$. For the experiments two different excitation frequencies were chosen: $f=500 \mathrm{~Hz}$ and $250 \mathrm{~Hz}$. During measurements the induced voltage changes caused by external tensile stress were detected. They were directly related to the changes of relative magnetic permeability tensor component parallel to the rod axis. In order to provide a methodological correctness the value of registered voltage was reduced by the value of separately measured "residual voltage" for the case, when there was no bar inside coils (which were distanced identically like in target experiment). Such procedure partially eliminated the problem of dispersion magnetic field present outside the bar around the magnetizing coil in quasi-open magnetic circuit (including magnetic elements of testing machine).

\section{Theoretical model}

A simple theoretical description of stress induced changes of magnetic permeability has been realized by modified, one-domain Stoner-Wohlfarth model. The density of longitudinal, stress-dependent anisotropy energy can be expressed as 


$$
E_{\sigma}(\theta)=-\frac{3}{2} \lambda_{s}\left(\sigma+\sigma_{i}\right) \sin ^{2} \theta
$$

where $\sigma$ and $\sigma_{i}$ are external and internal stresses, respectively, $\lambda_{s}$ is a saturation magnetostriction constant and $\theta$ denotes the angle between magnetization and direction perpendicular to rod axis. In order to reconstruct the consequences of realistic domain walls movements during $\mathrm{AC}$ magnetizing process and to consider the influence of mechanical stress the effective energy density of perpendicular anisotropy (PA) has been introduced: $E_{\text {eff }}(\sigma)=-K_{\text {eff }}(\sigma) \cos ^{2} \theta$, where:

$$
\begin{aligned}
& K_{\mathrm{eff}}(\sigma)=K_{p}+K_{\sigma}\left(\sigma+\sigma_{i}\right)^{2}+\delta\left[P_{1}\left(\frac{\sigma}{\sigma_{m}}-1\right)\right. \\
& \left.+P_{4}\left(\frac{\sigma}{\sigma_{m}}-1\right)^{4}+P_{5}\left(\frac{\sigma}{\sigma_{m}}-1\right)^{5}\right],
\end{aligned}
$$

in which $K_{p}$ is PA constant and $K_{\sigma}$ is a coefficient defining the strength of PA reversible changes described as square function of the tensile stress. The value of parameter $\delta$ is kept as 0 for increasing external stress, and it is set as 1, when stress is being decreased. This latter case corresponds to the irreversible changes of magnetic anisotropy both due to not perfect relaxation of internal stresses, as well as due to the hysteretic rearrangement of local magnetization directions. This modification of effective PA energy has been described phenomenologically using polynomial with first, fourth and fifth order terms and corresponding coefficients $P_{1}, P_{4}, P_{5}$ (the total stress has been related to the maximal external stress $\sigma_{m}$ applied during stretching). The high powers of the stress was introduced analogically in [11] for magnetomechanical modelling of coercive field.

Minimizing (over $\theta$ ) the sum of longitudinal and perpendicular anisotropy energy together with Zeeman energy of saturation magnetization $M_{s}$ in external magnetic field $H$ one calculates the magnetic permeability $\mu_{r}$, which is proportional to the field derivative of magnetization. The stress induced change of permeability related to the value for zero stress can be expresses with close formula:

$$
\frac{\Delta \mu_{r}(\sigma)}{\mu_{r}(\sigma=0, \delta=0)}=\frac{\alpha+\beta \sigma_{i}^{2}-\frac{3}{2} \sigma_{i}}{\frac{K_{\text {eff }}(\sigma)}{\lambda_{s}}-\frac{3}{2}\left(\sigma+\sigma_{i}\right)}-1,
$$

where $\alpha \equiv K_{p} / \lambda_{s}, \beta \equiv K_{\sigma} / \lambda_{s}$. For convenience of further analysis we also introduce new, reduced " $p$ " parameters: $p_{1} \equiv P_{1} / \lambda_{s}, p_{4} \equiv P_{4} / \lambda_{s}$ and $p_{5} \equiv P_{5} / \lambda_{s}$.

\section{Results and discussion}

Presented in Fig. 1 experimental results of relative $\mathrm{AC}$ magnetic permeability changes of rods for $f=500 \mathrm{~Hz}$ exhibit monotonic dependence on increasing tensile stress. The relative increase of $\mu_{r}$ for maximal stress (300 $\left.\mathrm{MPa}\right)$ is noticeable - almost 10\%, whereas the sensitivity of assembled magnetometric system allows to detect the changes smaller than $0.1 \%$. When gradually reducing the tensile stress, the magnetic permeability drops, nevertheless a huge hysteresis is observed. The obtained curves change from cycle to cycle, however the tendency to stabilization is visible (similarly to the changes of DC

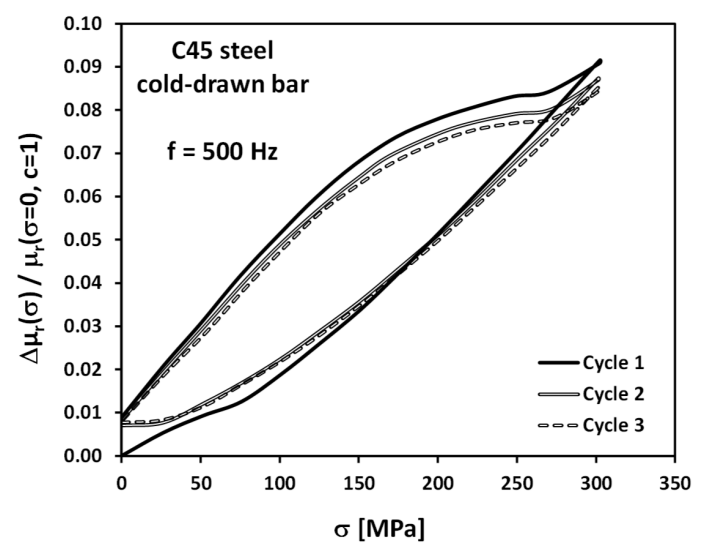

Fig. 1. Measured stress dependence of relative change of AC magnetic permeability for C45 steel cold-drawn bar $(f=500 \mathrm{~Hz}$, three cycles). For readability the spline interpolation curve is presented instead of experimental points.

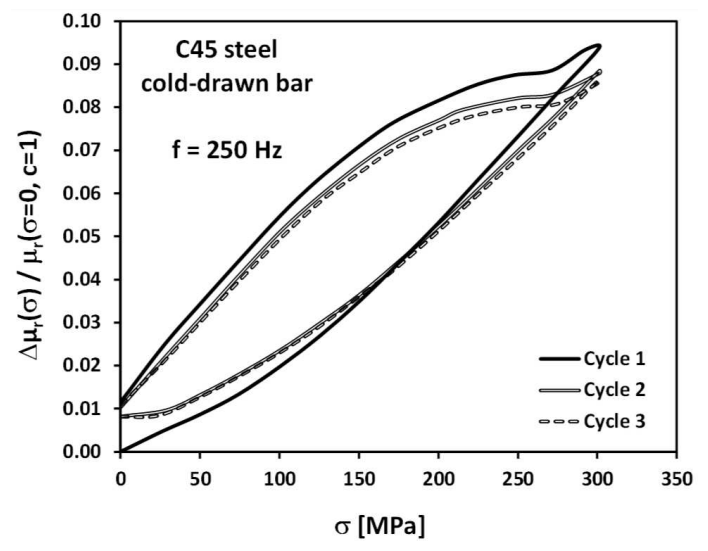

Fig. 2. Measured stress dependence of relative change of AC magnetic permeability for C45 steel cold-drawn bar $(f=250 \mathrm{~Hz}$, three cycles). For readability the spline interpolation curve is presented instead of experimental points.

magnetic parameters reported in historical articles [2, 3] for pure Fe and different kinds of steel). Almost the same results have been obtained for the frequency $f=250 \mathrm{~Hz}$ (Fig. 2). This fact convinces that both measurements have been done within low-frequency limit in which the role of eddy currents and the energy dissipation due to spin-lattice relaxation processes is negligible. However, the case of $f=500 \mathrm{~Hz}$ is preferable from technical point of view because of smaller noise amplitude to induced voltage value ratio. The observed magnitude of $\mathrm{AC} \mu_{r}$ relative changes remains in qualitative accordance with reported data [9] concerning Jiles-Atherton model DC parameters for C45 steel, however these parameters exhibit nonmonotonic behavior when stress exceeds Villari point (ca. $120 \mathrm{MPa}$ ).

The good quality fitting of theoretical model (3) to the experimental points is presented in Fig. 3 for C45 steel 


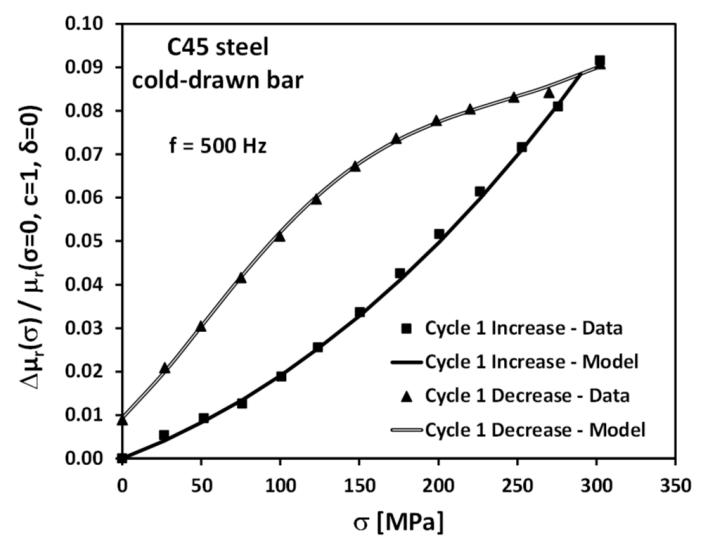

Fig. 3. Theoretical model (3) fitting to the experimental stress dependence of relative AC magnetic permeability change for C45 steel cold-drawn bar $(f=500 \mathrm{~Hz}$, $1^{\text {st }}$ cycle).

cold-drawn bar in the case of $f=500 \mathrm{~Hz}$ and the cycle $c=1$ (the normalized $\chi^{2}$ took low value - ca 1.1). The fitting parameters for all cycles are collected in Table I. Parameters $\alpha$ and $\beta$ have been found for $c=3, \delta=0$, $\sigma_{i}=0$ and fixed (under assumption that $K_{p}, K_{\sigma}$ and $\lambda_{s}$ quantities do not alter). The parameters $p_{1}, p_{4}$ and $p_{5}$ describing the magneto-mechanic hysteresis $(\delta=1)$, take very similar values for all cycles.

The significant changes are visible in the values of $\sigma_{i}$ interpreted as internal stress. Just for simplicity it has been assumed that for $c=3, \delta=0$ the value of internal stress is equal zero (as a reference point). In all cycles the value of $\sigma_{i}$ drops noticeably when passing from increasing external stress subcycle $(\delta=0)$ to decreasing case $(\delta=$ $1)$. For later cycles the parameter $\sigma_{i}$ alters towards less tensile or even to compressive internal stresses.

TABLE I

Fitting parameters of model (3) for $f=500 \mathrm{~Hz}(c-$ cycle number).

\begin{tabular}{|c|c|c|c|c|c|c|c|}
\hline \multirow{2}{*}{$c$} & \multirow{2}{*}{$\delta$} & \multirow{2}{*}{$\begin{array}{c}\alpha \\
{[\mathrm{GPa}]}\end{array}$} & \multirow{2}{*}{$\begin{array}{c}\beta \\
{[1 / \mathrm{GPa}]}\end{array}$} & \multirow{2}{*}{$\begin{array}{c}\sigma_{i} \\
{[\mathrm{MPa}]}\end{array}$} & $p_{1}$ & $p_{4}$ & $p_{5}$ \\
\hline & & & & & \multicolumn{3}{|c|}{ [GPa] } \\
\hline \multirow{2}{*}{1} & 0 & \multirow{6}{*}{15.5} & \multirow{6}{*}{-7.53} & 42.7 & - & - & - \\
\hline & 1 & & & 30.4 & 1.35 & 3.70 & 2.49 \\
\hline \multirow{2}{*}{2} & 0 & & & 4.9 & - & - & - \\
\hline & 1 & & & -3.5 & 1.18 & 3.64 & 2.47 \\
\hline \multirow{2}{*}{3} & 0 & & & 0.0 & - & - & - \\
\hline & 1 & & & -10.6 & 1.19 & 3.86 & 2.68 \\
\hline
\end{tabular}

\section{Conclusions}

Almost $10 \%$ change of $\mathrm{AC}$ magnetic permeability evoked by tensile stress was observed by means of designed magnetometric system as a plug-in for standard testing machine. The visible magneto-mechanical hysteresis can be described by simple theoretical model as a consequence both of stress irreversible changes of magnetic anisotropy (2) and internal stress relaxation $\sigma_{i}$.
Presented method of AC low-frequency magnetic permeability measurements seems to be an effective, nonexpensive way of monitoring the stress in large-sized mechanical elements made with steel - offering significant applicational potential. In future it is even intended to make the geometry of $\mathrm{AC}$ magnetometer more practical by placing the coils on the side of the bar. Moreover, the development of the theoretical description of stress dependent AC permeability would be recommended - based on stress-dependent, nonlinear constitutive relations proposed in very recent paper [12].

\section{Acknowledgments}

This work was supported in part by the University of Technology and Humanities in Radom under statutory grant $3179 / 25 / \mathrm{P}$ and by RADWAG Balances and Scales.

\section{References}

[1] E. Villari, Ann. Phys. Chem. 126, 87 (1865).

[2] J.A. Ewing, Magnetic induction in iron and other metals The Electrician Printing And Publishing Co, London 1900.

[3] M.F. Fischer, Bureau of Standards J. Res. 1, 721 (1928).

[4] I.M. Robertson, DSTO Materials Research Laboratory Technical Report MRL-TR-90-27, Maribyrnong 1991.

[5] K. Yaegashi, ISIJ Int. (Iron Steel Inst. Jpn.) 47, 327 (2007).

[6] A. Viana, L.-L. Rouve, G. Cauffet, J.-L. Coulomb, IEEE Trans. Magnet. 47, 1366 (2011).

[7] M. Witoś, M. Żokowski, Solid State Phenomena 220221, 544 (2015).

[8] A. Javed, T. Szumiata, N.A. Morley, M.R.J. Gibbs, Acta Materialia 58, 4003 (2010).

[9] D. Jackiewicz, R. Szewczyk, J. Salach, A. Bieńkowski, Acta Physica Polonica A 126, 392 (2014).

[10] D.C. Jiles, M.K. Devine, J. Appl. Phys. 76, 7015 (1994).

[11] R.C. Smith, M.J. Dapino, IEEE Trans. Magnet. 42 , 1944 (2006).

[12] P. Shi, K. Jin, X. Zheng, International Journal of Mechanical Sciences 124-125, 229 (2017). 